\title{
Effect of the extent of ethanol removal on the volatile compounds of a Chardonnay wine dealcoholized by vacuum distillation
}

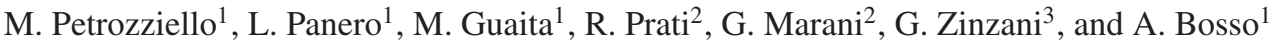 \\ ${ }^{1}$ Consiglio per la ricerca in agricoltura e l'analisi dell'economia agraria, Centro di Ricerca Viticoltura ed Enologia, Via Pietro Micca, \\ 35, 14100 Asti, Italy \\ ${ }^{2}$ CAVIRO DISTILLERIE via Convertite, 48018 Faenza, Italy \\ ${ }^{3}$ CAVIRO sca via Convertite, 48018 Faenza, Italy
}

\begin{abstract}
Beverages obtained from the partial dealcoholization of wine" are those drinks whose final alcoholic degree after dealcoholization is lower than that of a wine and higher than or equal to $0.5 \% \mathrm{v} / \mathrm{v}$. When the total alcoholic degree is lower than $0.5 \% \mathrm{v} / \mathrm{v}$ the denomination is "Beverages obtained from the dealcoholization of wine".

The practices to be authorized for the production of these drinks with the dealcoholized wine fractions are currently being studied at OIV. The characterization of the composition of these fractions is essential to identify the necessary corrective practices.

The present work was aimed at monitoring the losses of the main volatile compounds of a Chardonnay wine with the proceeding of the dealcoholization process by vacuum distillation. The wine was subjected to total dealcoholization, and during the process the evaporated fractions, re-condensed at $9{ }^{\circ} \mathrm{C}$, were collected in aliquots of $1.25 \mathrm{~L}$ each. The ethanol content of each fraction was measured, and for the first 20 fractions the content in volatile compounds was determined with GC-MS.

The results show that the losses of volatile compounds during the dealcoholization process follow different trends depending on the molecules considered. The most volatile compounds, generally with the lowest perception thresholds, were mainly present in the first evaporated fractions. The greatest losses concerned isoamylacetate, ethyl hexanoate and ethyl octanoate.

Conversely, a greater number of molecules were present at similar concentrations in the different fractions, and their losses followed a linear or sometimes exponential trend: in particular, these compounds included n-hexanol, 2-phenylethanol, diethyl succinate and medium chain fatty acids (hexanoic, octanoic and decanoic acids).

In the wine dealcoholized at $3.36 \% \mathrm{v} / \mathrm{v}$ (loss of ethanol equal to $7.43 \% \mathrm{v} / \mathrm{v}$, corresponding to the $20^{\text {th }}$ and last recondensed fraction), some volatile compounds were no longer detectable or quantifiable; in particular, these compounds were isoamylacetate, ethylhexanoate, hexylacetate, n-hexanol and other alcohols with 6 carbon atoms and ethyl octanoate.

Other compounds, such as hexanoic, octanoic and decanoic acids, and, in particolar, $\beta$-phenylethanol, benzylic aalcohol and $\gamma$-butyrolactone, underwent lower percentage losses than those of ethanol. The dealcoholization process can therefore deeply modify the original aromatic profile of the wines, intervening on the absolute concentration and on the relative ratios of the single molecules.
\end{abstract}

\section{Introduction}

The practice of dealcoholization can be performed by thermal distillation (under vacuum or not) and membrane technologies [1]. The most used thermal distillation processes are the spinning cone column and the distillation under vacuum.

The dealcoholization is used for the reduction of the alcohol content of wines to obtain dealcoholized drinks, which are distinguished in beverages obtained from the total or partial dealcoholization of the wine (OIV Resolutions OENO 432 and 433 - 2012), when their total alcohol content is respectively lower or higher than $0.5 \% \mathrm{v} / \mathrm{v}$.

After the treatment, the dealcoholized fractions are subjected to practices aimed at improving their sensory characteristics. In fact, if on the one hand the lower alcohol content can increase the volatility of some aromatic compounds [2-5], on the other hand the content in volatile compounds is significantly reduced during dealcoholization. Regarding the taste descriptors, the perception of heat decreases when reducing the alcohol content, and a loss of mouthfeel, balance and sweetness is perceived [6].

Studies have been carried out to verify the selectivity of the dealcoholization techniques on the composition of wines subjected to the removal of variable levels of ethanol. It was observed that the greatest losses concern the aromatic fraction [7-9] and that these losses are not uniform for all molecules [10-12]. 


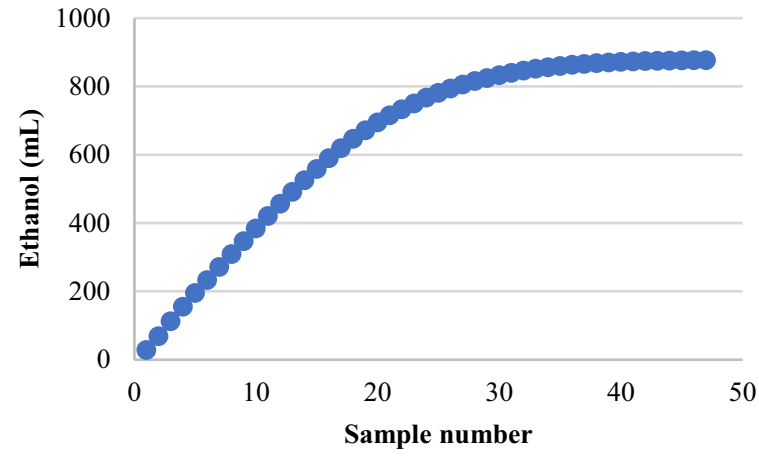

Figure 1. Losses of ethanol content during distillation.

On the contrary, as regards the fixed compounds (acids, salts, polyphenolic compounds), concentration increases are observed, which in most cases, in particular for the polyphenolic fraction, are proportional to the amount of solvent removed during the process (water and ethanol in the case of the distillation techniques, only ethanol for the membrane techniques) [12].

The practices to be authorized for the production of dealcoholized drinks are currently being studied at the OIV, and the knowledge of the composition of the dealcoholized fractions is fundamental to evaluate the necessary corrective measures.

The present work was focused on the monitoring of the removal of the main volatile compounds of a Chardonnay white wine with the progress of the dealcoholization process, carried out by distillation under vacuum.

\section{Materials and methods}

The total dealcoholization by vacuum distillation of $100 \mathrm{~L}$ Chardonnay was monitored. The process was performed with a vacuum evaporator (Reda Concentrator mod CM100, properly modified) that reaches the vacuum condition at a pressure of $30 \mathrm{mbar}$, with a working temperature ranging between 18 and $25^{\circ} \mathrm{C}$.

47 fractions of evaporated $(1.25 \mathrm{~L}$ each, for a total volume of $58.7 \mathrm{~L}$ ), recondensed at a temperature of about $9^{\circ} \mathrm{C}$, were collected continuously during the process. The most volatile fractions were recovered and condensed in a second exchanger at a temperature of $0-2{ }^{\circ} \mathrm{C}$ (fractions recondensed at $0{ }^{\circ} \mathrm{C}$ ). The sampling continued until the dealcoholization process was completed.

The ethanol content was measured for all dealcoholized fractions (official EEC method), and the content in volatile compounds was determined with GC-MS for the first 20 fractions, in duplicate.

\section{Results}

Figure 1 shows the trend of ethanol losses during the whole dealcoholization process determined by measuring the alcohol content of the 47 recondensed fractions recovered continuously until completion of the process.

The balance of the removals was obtained by adding the ethanol content of the 47 fractions to that of the additional 3 fractions recondensed at $0{ }^{\circ} \mathrm{C}$. The total ethanol removal amounted to $10.61 \% \mathrm{v} / \mathrm{v}$, and this result was consistent with the difference between the initial

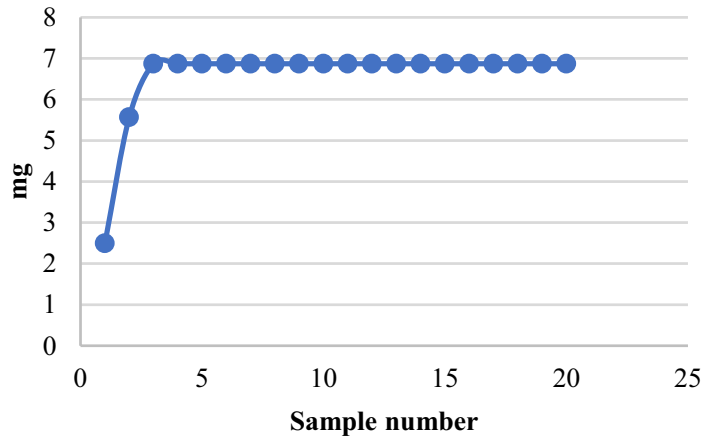

Figure 2. Isoamylacetate content in the gathered fractions.

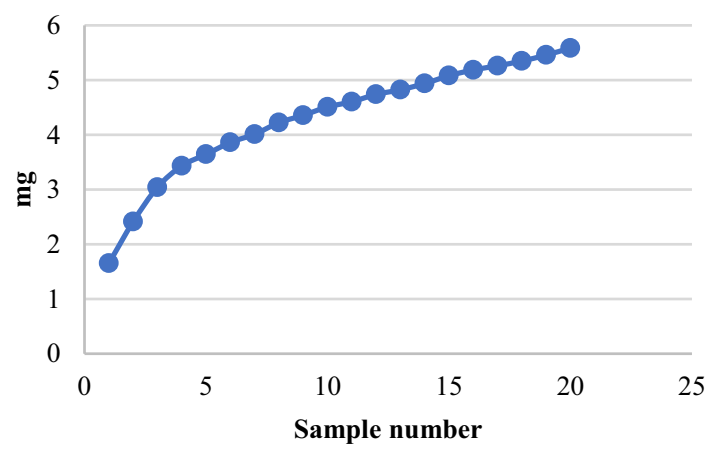

Figure 3. Ethylexanoate content in the gathered fractions.

alcohol content of the wine $(10.79 \% \mathrm{v} / \mathrm{v})$ and the final content of the dealcoholized product $(0.1 \% \mathrm{v} / \mathrm{v})$.

The monitoring of the losses in volatile substances concerned the first 20 evaporated/recondensed fractions, corresponding to a total volume of $25 \mathrm{~L}$.

23 different volatile compounds, mainly of fermentative and pre-fermentative origin, were identified and quantified: 5 ethyl esters (ethyl hexanoate, ethyl octanoate, ethyl lactate, diethyl succinate, monoethyl succinate), 3 acetate esters (hexyl acetate, isoamyl acetate, phenylethyl acetate), 7 alcohols (isobutanol, isoamyl alcohol, n-butanol, n-hexanol, cis-3-hexenol, $\beta$-phenylethanol, benzylic alcohol), 4 organic acids (isovaleric acid, hexanoic acid, octanoic acid, decanoic acid), as well as acetoin, $\gamma$-butyrolactone, 4-ethylphenol and ethyl-3-hydroxybutirate.

The losses trend of these compounds was measured during the dealcoholization process, and the total amount of the removals was measured in correspondence with the recovery of the $20^{\text {th }}$ evaporated/recondensed fraction.

As regards the losses trend, differences in behavior were observed between the analyzed molecules. Some molecules underwent consistent losses during the initial phase of the dealcoholization process (first 3-4 fractions), and a greater number of molecules showed an almost linear losses trend with the proceeding of the dealcoholization process.

The first group included isoamyl acetate (Fig. 2), ethyl hexanoate (Fig. 3), ethyl octanoate and hexyl acetate. In particular, isoamyl acetate was completely absent already in the fourth fraction, while the other esters showed a strong reduction in the slope of the losses trend between the $3^{\text {rd }}$ and $7^{\text {th }}$ fraction.

As regards the second group of molecules, the losses trend was perfectly linear for ethyl lactate, diethyl 


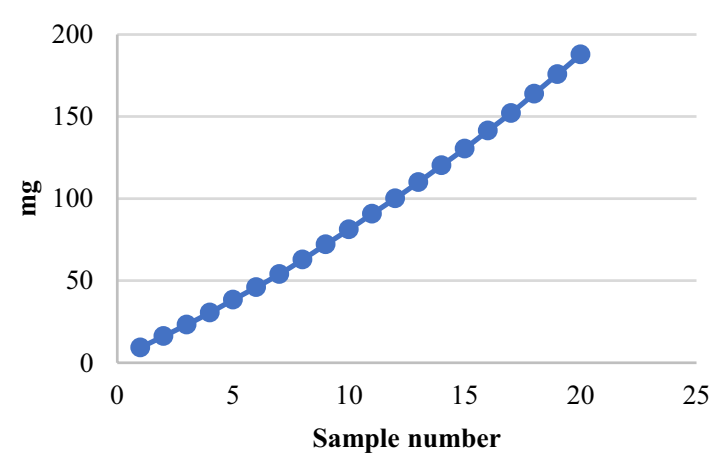

Figure 4. 2-phenylethanol content in the gathered fractions.

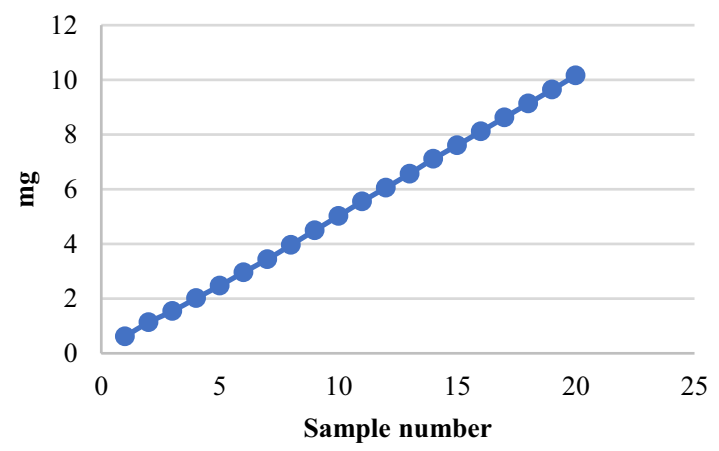

Figure 5. Isovalerianic acid content in the gathered fractions.

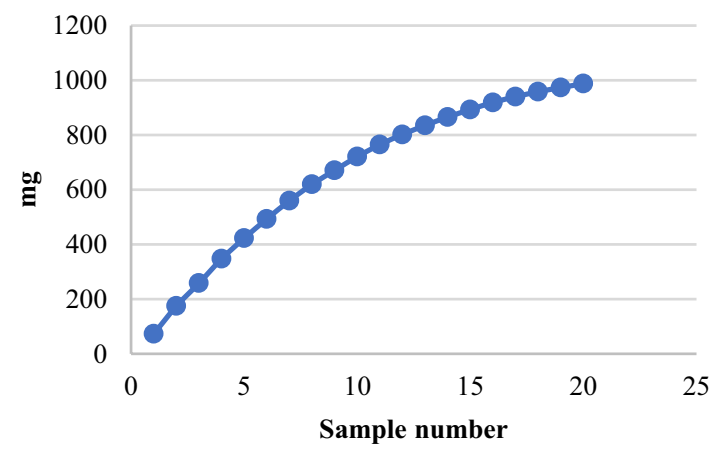

Figure 6. Isobutanol content in the gathered fractions.

succinate, benzylic alcohol, $\beta$-phenylethanol (Fig. 4), isovaleric acid (Fig. 5), hexanoic acid, octanoic acid, decanoic acid, 4-ethylphenol, acetoin, ethyl-3-hydroxy butirate and $\gamma$-butyrolactone, whereas for isobutanol (Fig. 6), isoamyl alcohol, n-butanol and n-hexanol and for cis-3-hexenol and phenylethyl acetate the trend tended to be logarithmic: the slope of the curve decreased in correspondence with the last fractions recovered.

Among the molecules quantified, only monoethylsuccinate was not detected in the fractions analyzed, with the exception of the last 3 fractions: this molecule recorded overall losses lower than $1 \%$ of the initial content.

The losses trend was influenced by the total extent of the removal of the different molecules. For each molecule analyzed, Table 1 reports the cumulative removal referred to the $20^{\text {th }}$ fraction recovered, and corresponding to a dealcoholisation level of $7.43 \% \mathrm{v} / \mathrm{v}$; the losses are expressed as a percentage of the initial content, also shown in the Table 1.

The molecules characterized by losses trends with high initial losses (isoamyl acetate, ethyl hexanoate, ethyl octanoate and hexyl acetate) were completely removed
Table 1. Chardonnay wine composition and cumulative removal percentage of the 23 volatile compounds referred to the $20^{\text {th }}$ fraction.

\begin{tabular}{|c|c|c|}
\hline & $\begin{array}{c}\text { Wine composition } \\
(\mathbf{m g} / \mathbf{L})\end{array}$ & $\begin{array}{c}\text { Removal } \\
(\boldsymbol{\%})\end{array}$ \\
\hline isobutanol & 14.28 & 106.7 \\
isoamly acetate & 1.71 & 79.7 \\
n-butanol & 0.69 & 91.4 \\
isoamyl alcohol & 159.92 & 150.3 \\
ethyl esanoate & 0.68 & 101.8 \\
hexyl acetate & 0.10 & 293.6 \\
acetoin & 0.34 & 103.6 \\
ethyl lactate & 10.32 & 23.8 \\
n-hexanol & 1.34 & 84.0 \\
cis-3-hexenol & 0.09 & 121.7 \\
ethyl octanoate & 0.50 & 102.1 \\
ethyl-3-idroxy-butirrate & 0.22 & 20.2 \\
$\gamma$-butirolactone & 5.09 & 4.2 \\
isovalerianic acid & 0.71 & 15.8 \\
diethylsuccinate & 1.57 & 18.5 \\
$\beta$-phenethyl acetate & 0.04 & 283.9 \\
esanoic acid & 1.92 & 21.9 \\
benzylic acid & 0.15 & 6.3 \\
$\beta$-phenylethanol & 27.10 & 7.6 \\
octanoic acid & 2.83 & 40.0 \\
4-ethylphenol & 0.35 & 10.4 \\
decanoic acid & 0.98 & 39.0 \\
monoethylsuccinate & 24.13 & 0.2 \\
\hline
\end{tabular}

from the dealcoholized wine. The $80 \%$ losses reported for isoamylacetate were not real: in fact (Fig. 2), from the $4^{\text {th }}$ fraction onwards isoamylacetate was absent (flat curve). This evidence probably depends on the fact that isoamylacetate, due to its high volatility, was not totally recovered in the fractions condensed at $9{ }^{\circ} \mathrm{C}$, and a great part was recovered in the additional fractions obtained at $0{ }^{\circ} \mathrm{C}$. $100 \%$ removals were also detected for isobutanol, nbutanol, n-hexanol, cis-3-hexenol and phenylethyl acetate, whose losses showed a linear trend and a final flattening of the curve, due to the imminent exhaustion.

As regards the molecules with perfectly linear losses trends, the removals were lower in percentage: 16-40\% for organic acids, $24 \%$ for ethyl lactate, $18 \%$ for diethyl succinate and $19 \%$ for 4-ethylphenol. Lower losses were observed for $\beta$-phenylethanol (7.6\%), benzylic alcohol $(6 \%)$ and $\gamma$ butyrolactone (4\%). These results agree with those of a previous work [12].

In accordance with the results obtained by some authors with red and rosé wines [7,10-12], we observed that the losses of $\beta$-phenylethanol and benzylic alcohol were modest even for a white wine, although it was hypothesized that the limited removals of these molecules could depend on the presence of $\pi-\pi$ interactions with the polyphenolic compounds, particularly abundant in red wines.

\section{Conclusions}

The losses trend of the volatile compounds of a Chardonnay wine was monitored during the dealcoholization process. Important differences between the molecules analyzed were observed as regards both the losses trend during the process and their total extent. The compounds that underwent the highest losses were the acetates of 
higher alcohols (in particular isoamylacetate), and the ethyl esters of medium chain fatty acids (ethylhexanoate and ethyl octanoate). The sensory profile of the wine is therefore influenced by the degree of dealcoholization achieved. On the basis of this result, in order to limit the losses of the most volatile compounds which are removed in high concentrations at the beginning of the dealcoholization process, it is possible to suggest that it could be more convenient to prolong the dealcoholization of a small fraction of wine that will be used in the blend with the wine to be corrected, rather than to process the whole mass of wine. However, this possibility is not currently allowed by the OIV regulation.

\section{References}

[1] L.M. Schmidtke, J.W. Blackman, S.O. Agboola, J. Food Sc. 77, R25 (2012)

[2] A.L. Robinson, S. Ebeler, H. Heymann, P.K. Boss, P.S. Solomon, R.D. Trengove, J. Agric. Food Chem. 57, 10313 (2009)

[3] M.C. Goldner, M.C. Zamora, P. Di Leo Lira, H. Gianninoto, A. Bandoni, J. Sensory Studies 24, 243 (2009)
[4] E.S. King, R.L. Dunn, H. Heymann, Food Qual. Prefer. 28, 235 (2013)

[5] B. Pineau, J.C. Barbe, C. Van Leeuwen, D. Dubourdieu, J. Agric. Food Chem. 57, 3702 (2009)

[6] S. Meillon, C. Urbano, G. Guillot, P. Schlich, Food Qual. Prefer 21, 763 (2010)

[7] N. Diban, V. Athes, M. Bes, I. Souchon, J. Membrane Sc. 311, 136 (2008)

[8] B. Fedrizzi, E. Nicolis, F. Camin, E. Bocca, C. Carbognin, M. Scholz, P. Barbieri, F. Finato, R. Ferrarini, Food Bioprocess Technol. 7, 62 (2014)

[9] E. Gómez-Plaza, J.M. López-Nicolás, J.M. LópezRoca, A. Martínez-Cutillas, LWT - Food Sc. Technol. 32, 384 (1999)

[10] L. Liguori, P. Russo, D. Albanese, M. Di Matteo, Food Chem. 140, 68 (2013)

[11] M.T. Lisanti, A. Gambuti, A. Genovese, P. Piombino, L. Moio, Food Bioprocess Technol. 6, 2298 (2013)

[12] S. Motta, M. Guaita, M. Petrozziello, A. Ciambotti, L. Panero, M. Solomita, A. Bosso, Food Chem. 221, 1 (2017) 